\title{
Front Matter: Volume 6952
}

, "Front Matter: Volume 6952," Proc. SPIE 6952, Laser Source Technology for Defense and Security IV, 695201 (29 April 2008); doi: 10.1117/12.800996

SPIE Event: SPIE Defense and Security Symposium, 2008, Orlando, Florida, SPIE. United States 


\section{PROCEEDINGS OF SPIE}

\section{Laser Source Technology for Defense and Security IV}

Mark Dubinskii

Gary L. Wood

Editors

17-18 March 2008

Orlando, Florida, USA

Sponsored and Published by

SPIE

Volume 6952 
The papers included in this volume were part of the technical conference cited on the cover and title page. Papers were selected and subject to review by the editors and conference program committee. Some conference presentations may not be available for publication. The papers published in these proceedings reflect the work and thoughts of the authors and are published herein as submitted. The publisher is not responsible for the validity of the information or for any outcomes resulting from reliance thereon.

Please use the following format to cite material from this book:

Author(s), "Title of Paper," in Laser Source Technology for Defense and Security IV, edited by Mark Dubinskii, Gary L. Wood, Proceedings of SPIE Vol. 6952 (SPIE, Bellingham, WA, 2008) Article CID Number.

ISSN 0277-786X

ISBN 9780819471437

Published by

SPIE

P.O. Box 10, Bellingham, Washington 98227-0010 USA

Telephone +1 3606763290 (Pacific Time) · Fax +1 3606471445

SPIE.org

Copyright (C 2008, Society of Photo-Optical Instrumentation Engineers

Copying of material in this book for internal or personal use, or for the internal or personal use of specific clients, beyond the fair use provisions granted by the U.S. Copyright Law is authorized by SPIE subject to payment of copying fees. The Transactional Reporting Service base fee for this volume is $\$ 18.00$ per article (or portion thereof), which should be paid directly to the Copyright Clearance Center (CCC), 222 Rosewood Drive, Danvers, MA 01923. Payment may also be made electronically through CCC Online at copyright.com. Other copying for republication, resale, advertising or promotion, or any form of systematic or multiple reproduction of any material in this book is prohibited except with permission in writing from the publisher. The CCC fee code is $0277-786 \mathrm{X} / 08 / \$ 18.00$.

Printed in the United States of America.

Publication of record for individual papers is online in the SPIE Digital Library.

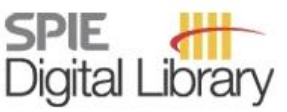

SPIEDigitallibrary.org

Paper Numbering: Proceedings of SPIE follow an e-First publication model, with papers published first online and then in print and on CD-ROM. Papers are published as they are submitted and meet publication criteria. A unique, consistent, permanent citation identifier (CID) number is assigned to each article at the time of the first publication. Utilization of CIDs allows articles to be fully citable as soon they are published online, and connects the same identifier to all online, print, and electronic versions of the publication. SPIE uses a six-digit CID article numbering system in which:

- The first four digits correspond to the SPIE volume number.

- The last two digits indicate publication order within the volume using a Base 36 numbering system employing both numerals and letters. These two-number sets start with 00, 01, 02, 03, 04, 05, $06,07,08,09,0 A, 0 B \ldots 0 Z$, followed by $10-12,20-2 Z$, etc.

The CID number appears on each page of the manuscript. The complete citation is used on the first page, and an abbreviated version on subsequent pages. Numbers in the index correspond to the last two digits of the six-digit CID number. 


\section{Contents}

vii Conference Committee

\section{SESSION 1 FIBER LASERS}

695202 Overview of Sandia's fiber laser program (Invited Paper) [6952-01]

D. A. V. Kliner, R. P. Bambha, B. T. Do, R. L. Farrow, Sandia National Labs. (USA); J.-P. Fève, JDSU (USA); B. P. Fox, Univ. of Arizona (USA); G. R. Hadley, A. Hansen, Sandia National Labs. (USA); H. J. Hoffman, M. Hotoleanu, Liekki Corp. (Finland); A. A. Hoops, W. L. Hsu, J. P. Koplow, Sandia National Labs. (USA); J. Koponen, Liekki Corp. (Finland); S. W. Moore, R. L. Schmitt, P. E. Schrader, Sandia National Labs. (USA); J. H. Simmons, K. Simmons-Potter, Univ. of Arizona (USA); A. V. Smith, Sandia National Labs. (USA); M. Söderlund, Liekki Corp. (Finland); W. J. Thomes, G. Wien, Sandia National Labs. (USA)

695204 Compact high-power eye safe fiber laser for LADAR [6952-03]

J. Henrie, M. S. Bowers, R. Afzal, B. Jenson, Aculight Corp. (USA); J. E. Grobmyer, U.S. Army Aviation and Missile Research, Redstone Arsenal (USA)

695205 Yb-free, SLM EDFA: comparison of 980-, 1470- and 1530-nm excitation for the core- and clad-pumping [6952-04] M. Dubinskii, Army Research Lab. (USA); V. Ter-Mikirtychev, NovaWave Technologies, Inc. (USA); J. Zhang, Army Research Lab. (USA); I. Kudryashov, Princeton Lightwave Inc. (USA)

695206 Parametric generation in optical fibers in the 900-950 nm spectral band [6952-05] W. Torruellas, M. Dennis, J. Warren, Johns Hopkins Univ. (USA); J. Hu, C. Menyuk, Univ. of Maryland, Baltimore County (USA)

695207 Comparison of spectral beam combining approaches for high power fiber laser systems [6952-06]

P. Madasamy, T. Loftus, A. Thomas, P. Jones, E. Honea, Aculight Corp. (USA)

\section{SESSION 2 DIODE LASERS}

695208 Advances in high-brightness semiconductor lasers [6952-07] M. L. Osowski, W. Hu, R. M. Lammert, S. W. Oh, P. T. Rudy, T. Stakelon, L. Vaissie, J. E. Ungar, QPC Lasers, Inc. (USA)

695209 Diode laser pumping sources for cryogenically cooled solid-state lasers [6952-08] M. A. Maiorov, I. E. Trofimov, Vullfort, Inc. (USA)

6952 OA High-brightness laser diode modules for $\mathrm{Yb}$ and Er fiber lasers [6952-09] M. A. Maiorov, I. E. Trofimov, Vullfort, Inc. (USA); C. Schnitzler, S. Hambücker, Ingeneric GmbH (Germany)

6952 OB High-power very high-brightness fiber-coupled diode laser arrays [6952-10]

D. M. Grasso, S. D. Roh, Coherent, Inc. (USA) 
6952 OC Mode control for high performance laser diode sources [6952-11]

P. Leisher, K. Price, S. Bashar, L. Bao, H. Huang, J. Wang, D. Wise, S. Zhang, S. Das,

M. DeFranza, A. Hodges, U. Trifan, D. Balsley, W. Dong, M. Grimshaw, M. DeVito, J. Bell,

R. Martinsen, J. Farmer, P. Crump, S. Patterson, nLight Corp. (USA)

6952 OD High power volume Bragg laser bar with 10 GHz spectral bandwidth [6952-12]

G. Venus, A. Gourevitch, College of Optics and Photonics, Univ. of Central Florida (USA);

V. Smirnov, OptiGrate (USA); L. Glebov, College of Optics and Photonics, Univ. of Central Florida (USA)

\section{SESSION 3 ADVANCED LASER CONCEPTS}

6952 OE A general model of a thermal conductivity for optical materials (Invited Paper) [6952-13] T. Taira, Y. Sato, Institute for Molecular Science (Japan)

6952 OG Single-frequency-mode Q-switched Nd:YAG laser controlled by volume Bragg gratings [6952-15]

N. Vorobiev, College of Optics and Photonics, Univ. of Central Florida (USA); V. Smirnov, OptiGrate (USA); L. Glebov, College of Optics and Photonics, Univ. of Central Florida (USA)

$6952 \mathrm{OH}$ Design and fabrication of efficient collimation and focusing optics for mid-IR quantum cascade lasers [6952-16]

B. E. Bernacki, K. Krishnaswami, N. C. Anheier, Jr., B. D. Cannon, Pacific Northwest National Lab. (USA)

$6952 \mathrm{Ol} \quad$ Cohering of multiple polariton lasers for sensing applications [6952-17]

R. L. Fork, L. A. Burgess, M. L. Davenport, D. M. Ramey, P. J. Reardon, D. B. Pollock,

R. G. Lindquist, D. M. Fork, Univ. of Alabama, Huntsville (USA)

\section{SESSION 4 HIGH POWER SSL}

6952 OK Kilowatt class high-power CW Yb:YAG cryogenic laser [6952-19]

D. C. Brown, J. M. Singley, E. Yager, K. Kowalewski, J. Guelzow, J. W. Kuper, Snake Creek Lasers, LLC (USA)

6952 OL 2.3-kW continuous operation cryogenic Yb:YAG laser [6952-37]

J. K. Brasseur, A. K. Abeeluck, A. R. Awtry, L. S. Meng, K. E. Shortoff, N. J. Miller,

R. K. Hampton, M. H. Cuchiara, D. K. Neumann, Directed Energy Solutions (USA)

$69520 \mathrm{M}$ Tensile strength and elastic moduli of composite solid state laser media [6952-21]

H.-C. Lee, H. E. Meissner, Onyx Optics Inc. (USA)

\section{SESSION 5 VISIBLE, EYE-SAFE, AND MID-IR LASERS}

$695200 \quad$ First laser performance of $\mathrm{Er}^{3+}$-doped scandia $\left(\mathrm{SC}_{2} \mathrm{O}_{3}\right)$ ceramic (Invited Paper) [6952-23] M. Dubinskii, N. Ter-Gabrielyan, L. D. Merkle, G. A. Newburgh, Army Research Lab. (USA); A. Ikesue, World Lab. Co., Ltd. (Japan) 
6952 OP Thermo-optical model for Er ${ }^{3+}:$ YAG gain media [6952-24]

M. Eichhorn, French-German Research Institute of Saint-Louis (France)

6952 OQ Design of walk-off corrected biaxial crystal composites [6952-25]

H.-C. Lee, H. E. Meissner, Onyx Optics Inc. (USA)

6952 OR Miniature solid-state lasers for pointing, illumination, and warning devices [6952-26]

D. C. Brown, J. M. Singley, E. Yager, K. Kowalewski, B. Lotito, J. Guelzow, J. Hildreth,

J. W. Kuper, Snake Creek Lasers, LLC (USA)

6952 OS Thulium fiber laser-pumped mid-IR OPO [6952-28]

D. Creeden, BAE Systems (USA); M. Jiang, Spectrode, LLC (USA); P. A. Budni,

P. A. Ketteridge, S. D. Setzler, Y. E. Young, J. C. McCarthy, P. G. Schunemann, T. M. Pollak, BAE Systems (USA); P. Tayebati, Spectrode, LLC (USA); E. P. Chicklis, BAE Systems (USA)

\section{SESSION 6 POSTER SESSION}

6952 OT Nonlinear optical device for middle infrared generation [6952-30]

N. B. Patel, Univ. of Campinas (Brazil)

6952 OU 300-kW eye-safe intracavity OPO transmitter [6952-31]

W. Zendzian, J. K. Jabczynski, J. Kwiatkowski, K. Kopczynski, Military Univ. of Technology (Poland)

$6952 \mathrm{OW}$ High-power diode lasers operating around 1500-nm for eyesafe applications [6952-35]

S. Patterson, P. Leisher, K. Price, K. Kennedy, W. Dong, M. Grimshaw, S. Zhang, J. Patterson,

S. Das, S. Karlsen, R. Martinsen, J. Bell, nLight Corp. (USA)

Author Index 
Downloaded From: https://www.spiedigitallibrary.org/conference-proceedings-of-spie on 26 Apr 2023

Terms of Use: https://www.spiedigitallibrary.org/terms-of-use 


\title{
Conference Committee
}

\author{
Symposium Chair \\ Larry B. Stotts, Defense Advanced Research Projects Agency (USA) \\ Symposium Cochair \\ Ray O. Johnson, Lockheed Martin Corporation (USA) \\ Program Track Chair
}

Gary W. Kamerman, FastMetrix, Inc. (USA)

Conference Chairs

Mark Dubinskii, Army Research Laboratory (USA)

Gary L. Wood, Army Research Laboratory (USA)

Program Committee

Steven R. Bowman, Naval Research Laboratory (USA)

Andrew J. W. Brown, Aculight Corporation (USA)

Joseph Mangano, Defense Advanced Research Projects Agency (USA)

Mark W. Neice, High Energy Laser Joint Technology Office (USA)

Stephen G. Post, Missile Defense Agency (USA)

\section{Session Chairs}

1 Fiber Lasers

Andrew J. W. Brown, Aculight Corporation (USA)

2 Diode Lasers

Steven R. Bowman, Naval Research Laboratory (USA)

3 Advanced Laser Concepts

Gary L. Wood, Army Research Laboratory (USA)

$4 \quad$ High Power SSL

Don D. Seeley, High Energy Laser Joint Technology Office (USA)

$5 \quad$ Visible, Eye-Safe, and Mid-IR Lasers

Stephen G. Post, Missile Defense Agency (USA) 
Downloaded From: https://www.spiedigitallibrary.org/conference-proceedings-of-spie on 26 Apr 2023

Terms of Use: https://www.spiedigitallibrary.org/terms-of-use 